\title{
Atenção odontológica durante a pandemia de COVID-19: uma revisão de literatura
}

\section{Dental attention during the COVID-19 pandemic: a literature review}

\author{
Lara Cristal Baldan' \\ Fabrício Farias Teixeira' iD \\ Thabata Cristy Zermiani" (iD
}

Secretaria de Saúde de Curitiba (SMS), Curitiba, PR, Brasil

" Pontifícia Universidade Católica do Paraná, Curitiba, PR, Brasil

\section{* E-mail: laracbaldan@gmail.com}

Recebido: 17 ago 2020

Aprovado: 16 set 2020

\section{RESUMO}

Introdução: 0 atendimento odontológico, em função da produção de aerossóis, traz riscos de contaminação cruzada, sendo essencial a adoção de estratégias preventivas. Em tempos de pandemia de COVID-19, tais cuidados foram revistos e ampliados. Objetivo: Analisar as estratégias de prevenção recomendadas para a atenção odontológica durante a pandemia da COVID-19. Método: Revisão integrativa por meio do acesso às bases de dados eletrônicas: PubMed, Cochrane Library, Scopus, Web of Science, SciELO, LILACS, MEDLINE via Biblioteca Virtual em Saúde (BVS) e à literatura cinzenta, por meio do Google Acadêmico. A análise das referências recuperadas foi realizada por dois revisores, para identificar estudos elegíveis com base nos títulos e resumos. Em seguida, foram obtidas as versões em texto completo dos artigos para leitura e extração de dados. Resultados: Dos 359 estudos obtidos a partir dos procedimentos de busca, 35 artigos foram incluídos nesta revisão. Observou-se a adoção de medidas preventivas prévias, concomitantes e posteriores ao atendimento odontológico, com maior destaque para as duas primeiras. Entre as medidas mais referidas, podem ser citadas: triagem do paciente; aferição de temperatura corporal; o atendimento somente de urgência e emergência; o uso de enxaguatório pré-procedimento; o uso de equipamentos de proteção individual, incluindo o respirador N95 ou FPP2/3; evitar a produção de aerossol; o cuidado na higiene das mãos; a desinfecção das superfícies, entre outros. Conclusões: Houve consenso no que se refere às medidas de prevenção da contaminação por SARS-CoV-2 nos estudos incluídos nesta revisão e entre estes e os protocolos gerados por instituições competentes. 0 conhecimento destas medidas, bem como sua aplicação prática, revela-se de grande valia para os cirurgiões-dentistas.

PALAVRAS-CHAVE: COVID-19; Pandemia; Odontologia; Prevenção de Doenças

\begin{abstract}
Introduction: Dental care, due to the production of aerosols, brings risks of cross contamination, and the adoption of preventive strategies is essential. In times of the COVID-19 pandemic, such care was reviewed and extended. Objective: To analyze the recommendations for dental care during a COVID-19 pandemic. Method: Integrative review through access to electronic databases PubMed, Cochrane Library, Scopus, Web of Science, SciELO, LILACS, MEDLINE in Virtual Health Library (VHL) and gray literature, through Google Scholar. The analysis of the retrieved references was carried out by two reviewers, to identify qualified studies based on the titles and abstracts. Then, they were written as full-text versions of the articles for reading and extracting data. Results: Of the 359 studies extracted from the procedures of search, 35 articles were included in this review. It was observed the adoption of preventive measures prior, concomitant and subsequent to dental care, with greater emphasis on the first two. Among the most basic measures, the following can be mentioned: patient screening; measurement of body temperature; urgent and emergency care only; the use of pre-procedure mouthwash; the use of Personal Protective Equipment, including the N95 or FPP2/3 respirator; avoiding the production of aerosol; care in hand hygiene; disinfecting surfaces, among others. Conclusions: There was a consensus regarding measures to prevent contamination by COVID-19 in the studies included in this review and between these and the results generated by institutions. Knowledge of these measures, as well as their practical application, is of great value to dentists.
\end{abstract}

KEYWORDS: COVID-19; Pandemic; Dentistry; Disease Prevention 


\section{INTRODUÇÃO}

Em 31 de dezembro de 2019, a Organização Mundial da Saúde (OMS) foi informada que, na cidade de Wuhan, província de Hubei, na República Popular da China, foram detectados casos de uma pneumonia de etiologia desconhecida ${ }^{1,2}$. Tratava-se de uma nova cepa de coronavírus que não havia sido identificada antes em seres humanos e que recebeu o nome de SARS-CoV-2. Posteriormente, em 30 de janeiro de 2020, foi declarado que o surto da doença causada pelo novo coronavírus, COVID-19, constituía uma Emergência de Saúde Pública de Importância Internacional e, no dia 11 de março de 2020, a COVID-19 foi caracterizada pela OMS como uma pandemia².

O novo coronavírus apresenta uma alta taxa de infecção, porém uma taxa de letalidade relativamente baixa ${ }^{4}$. Segundo dados do Ministério da Saúde, o Brasil apresenta taxa de letalidade de $4,5 \%$, podendo ser maior em idosos e pacientes com outras comorbidades $^{3,4}$. Os sintomas podem variar de um resfriado até uma pneumonia severa, sendo os mais comuns: febre, tosse seca ou secretiva, dor de garganta e falta de ar. Podem ocorrer também: cansaço, coriza, dores de cabeça, náuseas, vômito, diarreia, perda de olfato e paladar, dores musculares e calafrios ${ }^{5,6}$.

As principais vias de transmissão do SARS-CoV-2 incluem transmissão direta através de tosse, espirro e perdigotos, além da transmissão por contato com mucosa oral, nasal e ocular após superfícies contaminadas serem tocadas ${ }^{4,7}$. 0 aerossol também é uma possível via de transmissão, principalmente quando o vírus estiver em alta concentração e esse estiver ocorrendo em ambientes fechados. Desta forma, procedimentos odontológicos de rotina são um risco potencial para os profissionais e os pacientes ${ }^{8}$.

A prática odontológica envolve o uso de instrumentos odontológicos e cirúrgicos rotativos, como peças de mão, ultrassons e seringas de ar e água. Esses instrumentos criam um spray invisível que contém gotículas de água, saliva, sangue e microrganismos, os quais podem permanecer viáveis e sobreviverem por até 3 dias em superfícies inanimadas à temperatura ambiente, com maior preferência por condições de umidade ${ }^{7,9}$.

A melhor maneira de prevenir doenças é adotar ações para impedir a propagação dos seus agentes etiológicos. Por esse motivo, o controle de ambientes com risco biológico é parte da rotina e do conhecimento de todos os profissionais da área da Odontologia $^{10}$. Certos cuidados devem ser ainda mais rigorosos na prática odontológica para proteger a equipe e os pacientes, como: cuidados na sala de espera e na sala de atendimento, limpeza das superfícies, desinfecção de equipamentos e instrumentais, uso de equipamentos de proteção individual (EPI), lavagem de mãos, entre outros ${ }^{11,12}$.

Desde o início da pandemia, várias instituições mundiais se pronunciaram em relação às mudanças na rotina do atendimento odontológico, para evitar o contágio dos profissionais e a possível contaminação cruzada entre os pacientes. Instituições essas como Centers for Disease Control and Prevention (CDC $)^{7}$ e American Dental Association (ADA) ${ }^{13}$; em âmbito nacional, a
Agência Nacional de Vigilância Sanitária (Anvisa) ${ }^{14}$, o Conselho Federal de Odontologia (CFO) ${ }^{15}$ e a Associação de Medicina Intensiva Brasileira (AMIB) ${ }^{11}$.

Por conta dos vários protocolos publicados até o momento em todo o mundo e no Brasil, faz-se necessário sintetizar os principais aspectos abordados no momento para que os profissionais os apliquem em sua rotina diária. 0 objetivo desse estudo, portanto, foi sistematizar as principais estratégias adotadas na atenção odontológica durante a pandemia de COVID-19, descritas na literatura científica, para prevenir a contaminação cruzada.

\section{MÉTODO}

Esta revisão integrativa se fundamentou na seguinte pergunta de pesquisa: "Quais estratégias preventivas mais recomendadas na literatura científica devem ser adotadas na atenção odontológica durante a pandemia de COVID-19?". A revisão foi realizada por meio do acesso às bases de dados eletrônicas PubMed, Cochrane Library, Scopus, Web of Science, Scientific Electronic Library Online (SciELO), Literatura Latino-Americana e do Caribe em Ciências da Saúde (LILACS) e MEDLINE via Biblioteca Virtual em Saúde (BVS). Houve também a exploração da literatura cinzenta, usando o Google Acadêmico. As buscas foram realizadas no dia 10 de junho de 2020. Os descritores empregados na estratégia de busca foram: ("SARS-CoV-2" OR "COVID-19") AND (Dentist OR Dentistry).

Foram definidos como critérios de inclusão: I) os estudos que abordassem a temática dos riscos de infecção cruzada no atendimento odontológico durante a pandemia de COVID-19 e os cuidados necessários para evitá-los; II) estudos que descrevessem protocolos de atendimento durante a pandemia de COVID-19; III) estudos publicados em inglês, espanhol ou português nos anos de 2019 e 2020. Como critérios de exclusão foram considerados: I) estudos cuja ênfase não era dada aos cuidados durante o atendimento odontológico em tempos de pandemia de COVID-19; II) cartas, editoriais e comunicação breve.

Os estudos recuperados foram importados para um gerenciador de referências (Endnote). As duplicatas foram removidas e uma triagem inicial dos títulos e resumos foi realizada por dois revisores independentes, de acordo com os critérios de inclusão/exclusão. 0 teste de concordância Kappa foi conduzido ao fim desta etapa, chegando a um valor de $\mathrm{k}=0,72$, havendo, portanto, concordância substancial entre os revisores, segundo a classificação de Landis e Koch ${ }^{16}$.

Após a análise dos resumos, todos os artigos selecionados foram obtidos na íntegra e, posteriormente, examinados de acordo com os critérios de inclusão estabelecidos. Quando havia discordâncias na decisão final sobre um determinado artigo, estas foram discutidas a fim de obter consenso. Utilizou-se uma tabela de extração para especificar as características da amostra, o desenho do estudo, o país em que foi conduzido e para detalhar os cuidados odontológicos citados nos artigos. 
A fim de fazer uma síntese sobre os achados na literatura, foi elaborado um gráfico de bolhas acerca das estratégias preventivas adotadas frente ao atendimento odontológico. Estas foram categorizadas de acordo com o momento em que devem ser adotadas (antes, durante ou após o atendimento), sendo representadas por diferentes cores. Além disso, as bolhas assumiram diferentes diâmetros, de acordo com o número de artigos que as citaram.

\section{RESULTADOS}

As estratégias de busca e seus resultados encontram-se descritos na Figura 1. Inicialmente foram encontrados 338 estudos nas bases de dados informadas e 21 na literatura cinzenta. Após a remoção das duplicatas, análise de títulos, resumos e leitura de texto completo, quando pertinente, restaram 35 estudos para inclusão.
Na Quadro 1, encontram-se descritas as características dos estudos incluídos, como: autor, país, desenho do estudo e objetivo. Nos artigos analisados e selecionados neste estudo, há predominância de estudos conduzidos no continente americano $^{4,9,17,18,19,20,21,22,23,24,25,26,27,28}$.

Quanto ao desenho de estudo, as revisões de literatura foram as mais frequentes $4,8,9,17,18,19,20,21,22,23,24,25,26,27,28,29,30,31,32,33,34,35,36,37,38,39,40,41,42,43$. Apenas quatro estudos foram classificados como pesquisas quantitativas transversais ${ }^{44,45,46,47}$.

Na Figura 2 estão especificadas as principais estratégias preventivas sugeridas nos artigos, as quais foram divididas em antes, durante e após o atendimento odontológico.

Em se tratando das estratégias anteriores ao atendimento, emergiram as seguintes categorias: triagem prévia ${ }^{4,8,9,17,19,20,21,22,23,}$ $25,26,27,28,29,30,31,32,33,34,35,36,37,38,39,40,41,42,43,47,48$, cuidados na sala

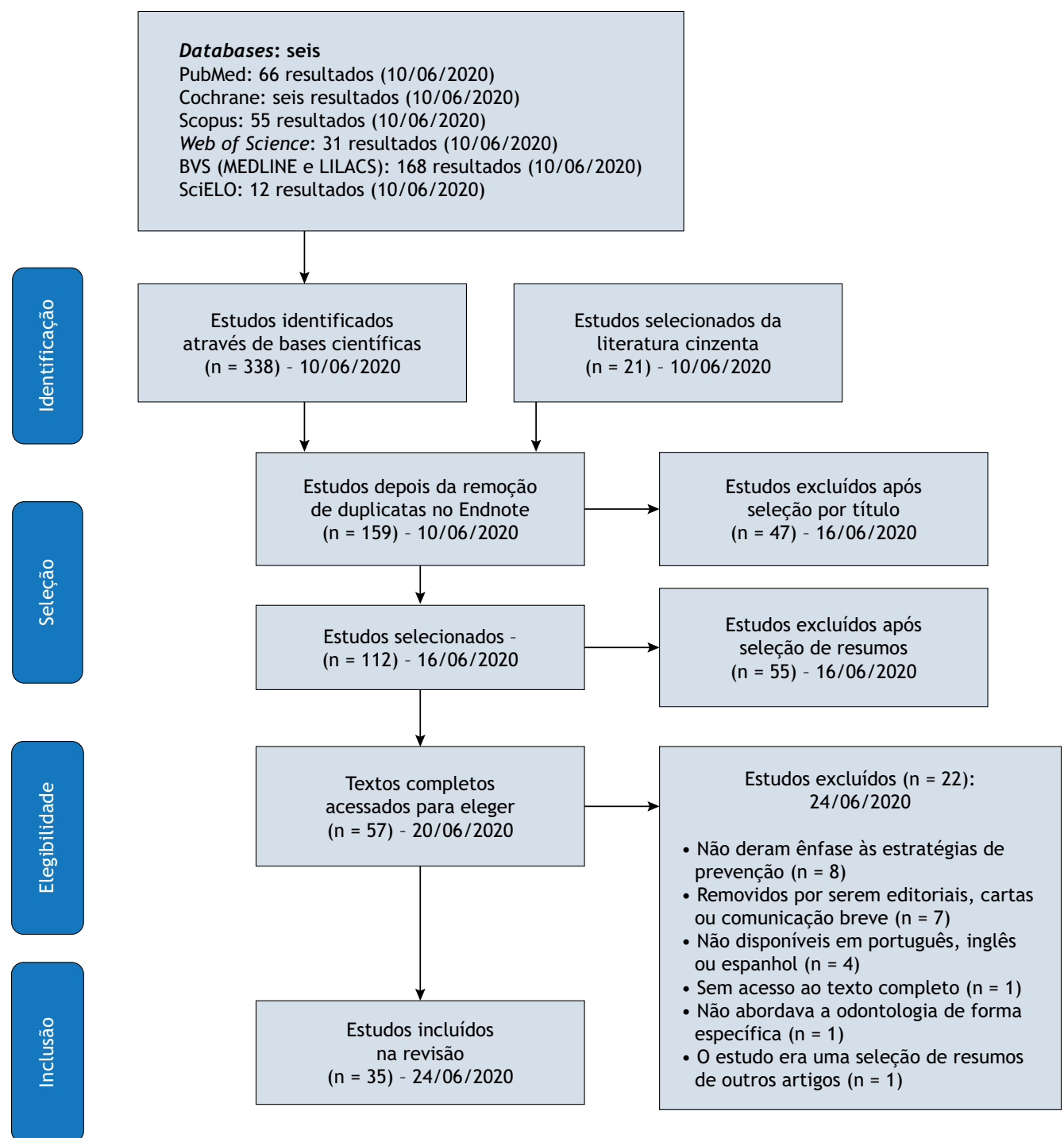

Fonte: Elaborada pelos autores, 2020.

Figura 1. Fluxograma acerca do processo de seleção dos estudos. 
Quadro 1. Artigos incluídos na revisão e suas características de acordo com país de publicação, desenho de estudo e objetivo.

\begin{tabular}{|c|c|c|c|}
\hline Autor & País & Desenho & Objetivo \\
\hline Araya-Salas ${ }^{28}$ & Chile & Revisão de literatura & $\begin{array}{l}\text { Apresentar várias considerações para o atendimento odontológico, a fim de reduzir o risco } \\
\text { ao vírus. }\end{array}$ \\
\hline Ather et al. ${ }^{9}$ & EUA & Revisão de literatura & Fornecer uma breve visão geral da epidemiologia, sintomas e vias de transmissão da doença. \\
\hline Bizzoca et al. ${ }^{43}$ & Itália & $\begin{array}{l}\text { Revisão de literatura } \\
\text { narrativa }\end{array}$ & $\begin{array}{l}\text { Construir protocolos endereçados aos dentistas, avaliar e modular os riscos de contágio } \\
\text { na odontologia. }\end{array}$ \\
\hline Cagetti et al. ${ }^{47}$ & Itália & $\begin{array}{l}\text { Estudo quantitativo } \\
\text { transversal }\end{array}$ & $\begin{array}{l}\text { Avaliar sinais/sintomas, medidas de proteção, nível de conscientização e a percepção sobre } \\
\text { o surto de COVID-19 entre dentistas que trabalham no norte da Itália. }\end{array}$ \\
\hline Careddu et al. ${ }^{42}$ & Irlanda & Revisão de literatura & $\begin{array}{l}\text { Coletar literatura disponível e fornecer acesso com base em publicações e guias sobre as } \\
\text { epidemias de SARS e MERS e informações disponíveis até o momento de SARS-CoV- } 2 \text {. }\end{array}$ \\
\hline Cirillo ${ }^{41}$ & Austrália & Revisão de literatura & $\begin{array}{l}\text { Coletar evidências e recursos atualmente disponíveis para ajudar os profissionais de } \\
\text { odontologia a minimizar a propagação de SARS-CoV-2. }\end{array}$ \\
\hline Duruk et al. ${ }^{46}$ & Turquia & $\begin{array}{l}\text { Estudo quantitativo } \\
\text { transversal }\end{array}$ & $\begin{array}{l}\text { Investigar que tipo de precauções os dentistas turcos tomam em clínicas odontológicas } \\
\text { durante a pandemia de COVID-19. }\end{array}$ \\
\hline Fallahi et al. ${ }^{40}$ & Irã & Revisão de literatura & $\begin{array}{l}\text { Fornecer um protocolo abrangente para gerenciar a possível exposição a pacientes ou } \\
\text { aqueles suspeitos de ter a COVID-19. }\end{array}$ \\
\hline Fini $^{27}$ & EUA & Revisão de literatura & $\begin{array}{l}\text { Coletar todas as informações necessárias para os dentistas sobre a pandemia de } \\
\text { COVID-19 em todo o mundo. }\end{array}$ \\
\hline Franco et al. ${ }^{25}$ & Brasil & Revisão de literatura & $\begin{array}{l}\text { Direcionar os conceitos apresentados na literatura mundial sobre biossegurança e EPI, para } \\
\text { sistematizar as recomendações para a prática clínica do atendimento odontológico durante } \\
\text { a pandemia de COVID-19. }\end{array}$ \\
\hline Gambhir et al. ${ }^{44}$ & Índia & $\begin{array}{l}\text { Estudo quantitativo } \\
\text { transversal }\end{array}$ & $\begin{array}{l}\text { Avaliar as práticas de conhecimento, conscientização e higiene em relação à } \\
\text { COVID-19 entre dentistas particulares de Tricity na Índia durante esses momentos críticos. }\end{array}$ \\
\hline Ge et al. ${ }^{8}$ & China & Revisão de literatura & $\begin{array}{l}\text { Compreender o significado da transmissão de aerossóis e sua implicação na odontologia para } \\
\text { facilitar a identificação e a correção de negligência na prática odontológica diária. Além de } \\
\text { levantar algumas precauções especiais que devem ser implementadas durante o surto. }\end{array}$ \\
\hline Giudice $^{37}$ & Itália & Revisão de literatura & $\begin{array}{l}\text { Discutir e sugerir os procedimentos mais adequados em todos os aspectos da prática } \\
\text { odontológica para reduzir o risco de infecção. }\end{array}$ \\
\hline Izzetti et al. ${ }^{39}$ & Itália & Revisão de literatura & $\begin{array}{l}\text { Discutir os riscos relacionados à prática odontológica e as recomendações atuais para } \\
\text { os dentistas. }\end{array}$ \\
\hline Jamal et al. ${ }^{38}$ & $\begin{array}{l}\text { Emirados } \\
\text { Árabes }\end{array}$ & Revisão de literatura & $\begin{array}{l}\text { Fornecer uma revisão das diretrizes e recomendações para limitar a transmissão do } \\
\text { SARS-CoV-2 para dentistas. }\end{array}$ \\
\hline Khader et al. ${ }^{45}$ & Jordânia & $\begin{array}{l}\text { Estudo quantitativo } \\
\text { transversal }\end{array}$ & $\begin{array}{l}\text { Avaliar o nível de conscientização, percepção e atitude em relação à doença pelo } \\
\text { coronavírus em dentistas jordanianos. }\end{array}$ \\
\hline Long et al. ${ }^{36}$ & Georgia & Revisão de literatura & $\begin{array}{l}\text { Apresentar revisões e protocolos implementados por diretores e residentes no Dental } \\
\text { College da Geórgia para gerenciar uma clínica de emergência odontológica durante a } \\
\text { pandemia do COVID-19. }\end{array}$ \\
\hline Maia et al. ${ }^{24}$ & Brasil & Revisão de literatura & $\begin{array}{l}\text { Sistematizar a produção bibliográfica sobre as recomendações, práticas e cuidados } \\
\text { adotados no atendimento odontológico em tempos de COVID-19, assim como, propor um } \\
\text { protocolo de atendimento odontológico nas unidades de saúde bucal da Polícia Militar do } \\
\text { estado do Rio de Janeiro. }\end{array}$ \\
\hline $\begin{array}{l}\text { Martins- } \\
\text { Chaves et al. }{ }^{23}\end{array}$ & Brasil & Revisão de literatura & $\begin{array}{l}\text { Revisar a literatura e discutir questões imunológicas relacionadas à COVID-19. Também } \\
\text { foram feitas sugestões para o apoio de pacientes imunocomprometidos neste novo contexto } \\
\text { emergente da prática clínica odontológica. }\end{array}$ \\
\hline Meng et al. ${ }^{35}$ & China & Revisão de literatura & $\begin{array}{l}\text { Apresentar o conhecimento essencial sobre COVID-19 e infecção hospitalar em ambientes } \\
\text { odontológicos e fornecer protocolos de gerenciamento recomendados para dentistas e } \\
\text { estudantes em áreas (potencialmente) afetadas. }\end{array}$ \\
\hline Passarelli et al. ${ }^{34}$ & Itália & Revisão de literatura & $\begin{array}{l}\text { Propor um breve questionário e um fluxograma para definir o risco que cada paciente } \\
\text { acarreta e adaptar adequadamente cada procedimento com base no risco do paciente. }\end{array}$ \\
\hline Peditto et al. ${ }^{48}$ & Itália & Relato de experiência & $\begin{array}{l}\text { Apresentar um fluxo de trabalho para gerenciar procedimentos odontológicos já em uso na } \\
\text { unidade de Odontologia do HU de Messina. }\end{array}$ \\
\hline Peng et al. ${ }^{33}$ & China & Revisão de literatura & $\begin{array}{l}\text { Resumir as possíveis rotas de transmissão de } 2019-n C o v \text {, e sua propagação. Além de revisar } \\
\text { as estratégias práticas para bloquear a transmissão do vírus durante o diagnóstico e } \\
\text { tratamento odontológico. }\end{array}$ \\
\hline Pereira et al. ${ }^{22}$ & Brasil & Revisão de literatura & $\begin{array}{l}\text { Esclarecer os dentistas sobre a história e a microbiologia do vírus, além de orientar sobre } \\
\text { como proceder durante as consultas de emergência com base em documentos internacionais. }\end{array}$ \\
\hline Pinto et al. ${ }^{21}$ & Brasil & Revisão de literatura & $\begin{array}{l}\text { Descrever as recomendações necessárias no atendimento de pacientes em clínicas } \\
\text { odontológicas em meio à pandemia de COVID-19. }\end{array}$ \\
\hline Ren et al. ${ }^{20}$ & EUA & Revisão de literatura & $\begin{array}{l}\text { Ajudar os profissionais de odontologia a entender melhor os riscos da transmissão de } \\
\text { doenças em ambientes dentários. }\end{array}$ \\
\hline Sales et al. ${ }^{19}$ & Brasil & Revisão de literatura & $\begin{array}{l}\text { Estabelecer condutas que possam reduzir a contaminação pelo SARS-CoV-2 entre a população } \\
\text { e a equipe odontológica durante o atendimento no sistema público e privado de saúde. }\end{array}$ \\
\hline $\begin{array}{l}\text { Sepúlveda- } \\
\text { Verdugo et al. }{ }^{18}\end{array}$ & Chile & Revisão de literatura & $\begin{array}{l}\text { Fornecer recomendações atualizadas e relevantes para a nossa realidade nacional, a fim de } \\
\text { reduzir as chances de contágio diante da exposição iminente de pacientes com suspeita ou } \\
\text { quem possam apresentar COVID-19. }\end{array}$ \\
\hline
\end{tabular}


Continuação

\begin{tabular}{|c|c|c|c|}
\hline Autor & País & Desenho & Objetivo \\
\hline $\begin{array}{l}\text { Shamszadeh } \\
\text { et al. }{ }^{32}\end{array}$ & Irã & Revisão de literatura & $\begin{array}{l}\text { Revisar o conhecimento básico sobre COVID-19 e abordar os protocolos recomendados de } \\
\text { controle de infecção para os dentistas e tratamentos em áreas hipoteticamente afetadas. }\end{array}$ \\
\hline Silva et al. ${ }^{26}$ & Brasil & $\begin{array}{l}\text { Pesquisa documental e } \\
\text { revisão de literatura }\end{array}$ & $\begin{array}{l}\text { Analisar os protocolos odontológicos disponibilizados nos países do Mercosul, apontando as } \\
\text { principais semelhanças e discrepâncias entre eles. }\end{array}$ \\
\hline Tuñas et al. ${ }^{4}$ & Brasil & Revisão de literatura & $\begin{array}{l}\text { Apresentar características clínicas da doença COVID-19, vias de transmissão, apresentar } \\
\text { medidas preventivas para controlar e minimizar a infecção no serviço odontológico. }\end{array}$ \\
\hline Turkistani ${ }^{31}$ & $\begin{array}{l}\text { Arábia } \\
\text { Saudita }\end{array}$ & Revisão de literatura & $\begin{array}{l}\text { Relatar aos ortodontistas o surgimento, a epidemiologia, os riscos e as precauções durante } \\
\text { a crise da doença. }\end{array}$ \\
\hline Volgenant et al. ${ }^{30}$ & Holanda & Revisão de literatura & $\begin{array}{l}\text { Resume medidas de controle de infecção para o ambiente de assistência odontológica em } \\
\text { relação ao SARS-CoV-2, com base nas evidências científicas atualmente disponíveis. }\end{array}$ \\
\hline Wu et al. ${ }^{29}$ & Canadá & Revisão de literatura & $\begin{array}{l}\text { Compartilhar as perspectivas de dentistas que praticam em consultório particular e clínicos } \\
\text { pesquisadores em instituições acadêmicas de odontologia. }\end{array}$ \\
\hline Xavier et al. ${ }^{17}$ & Brasil & Revisão de literatura & $\begin{array}{l}\text { Esclarecer a importância das práticas de CTBMF e o manejo clínico, utilizando como base a } \\
\text { residência em CTBMF no HU de Belém, no atendimento dos pacientes durante a pandemia } \\
\text { do coronavírus. }\end{array}$ \\
\hline
\end{tabular}

Fonte: Elaborado pelos autores, 2020.

EUA: Estados Unidos da América; SARS: síndrome respiratória aguda grave; MERS: síndrome respiratória do Oriente Médio; Mercosul: Mercado Comum do Sul; EPI: equipamento de proteção individual; HU: hospital universitário; CTBMF: Cirurgia e traumatologia bucomaxilofacial.

de medidas de proteção para 0 paciente ${ }^{4,8}$, $9,17,18,19,21,22,23,24,25,26,27,28,29,30,31,32,33,34,35,36,37,38,39,40,41,42,43,45,46,47,48 \quad$ e outros cuidados preventivos $4,8,9,17,18,19,20,21,22,23,24,25,26,27,28,29,30,31,32,35$, $36,37,38,39,40,41,42,43,44,46,48$.

Foram estabelecidos como estratégias de triagem prévia: a triagem presencial $4,8,9,17,19,20,23,25,27,28,30,31,32,33,35,36,38,39,40,41,47,48, \quad$ o contato telefônico $9,21,22,25,26,27,29,30,34,36,37,38,39,41,42,43,47,48$ e o encaminhamento de pacientes sintomáticos para atendimento médico $4,20,21,31,32,33,35$. Os cuidados na sala de espera foram bastante $\quad$ citados $^{4,8,9,21,22,23,24,25,26,27,29,30,31,32,34,35,37,38,39,40,41,42,43,45,47,48}$, incluindo: a organização do fluxo de pacientes por meio de agendamento $21,22,23,25,26,29,30,31,34,35,37,38,39,41,43,47,48$, a ventilação $8,9,21,27,30,31,32,37,39,41,42,45,47,48, \quad 0$ distanciamento de pacientes $8,23,24,27,29,31,32, \quad 34,37,38,41,43,45,47$, a remoção de obje$\operatorname{tos}^{4,21,25,29,30,37,38,39,43}$, a separação de casos suspeitos $4,9,27,40,41,42,45 \mathrm{e}$ a higiene das superfícies ${ }^{25,38,48}$.

Com relação às medidas de proteção para o paciente $e^{4,8,9}$, $17,18,19,21,22,23,24,25,26,27,28,29,30,31,32,33,34,35,36,37,38,39,40,41,42,43,45,46,47,48$, foram preconizados: o uso de enxaguatório pré-procedimento $^{4,8,9,18,19,20,22,23,24,26,27,28,29,30,31,32,33,34,35,36,37,38,39,40,41,42,43,46,47,48}$, a aferição de temperatura $4,8,9,19,21,23,25,27,28,31,32,33,34,35,36,38,39,40,41,47,48$, o uso de EPI pelos pacientes $4,9,21,22,23,25,27,32,34,35,36,38,40,41,42,45,47,48$, a higienização das mãos com água e sabão e/ou álcool ${ }^{4,21}$, $23,24,25,28,32,34,37,39,41,42,43,45,47,48$, a orientação de etiqueta respiratória $^{4,8,9,17,18,25,26,27,29,32,37,38,41,42}$, a especificação de atendimentos/ autoquarentena para pacientes confirmados/suspeitos/sintomáticos $20,23,27,30,31,33,35,36,40,42$, a especificação de atendimento de pacientes de risco $23,26,34,37,47$, os cuidados com objetos pessoais $^{37,39,47,48}$ e a antissepsia extraoral ${ }^{24,25,26}$.

Outros cuidados preventivos citados foram: a priorização do atendimento de urgências e emergências $^{8,9,17,18,19,20,21,22,24,25,26,28,29,30,32,35,36,37,38,39,40,41,42,44,46,48}$, a abordagem farmacêutica $9,17,18,22,23,27,29,30,38,48$, o atendimento de todos os pacientes como se fossem positivos para o vírus $4,9,20,22,25,36,43$, evitar acompanhantes ${ }^{25,29,37,38,39,41,43}$, o cuidado com a equipe odontológica $^{4,25,31,36,37,40,48}$ e a disponibilização de kit do teste rápido ${ }^{20,30,36}$.

No que se refere às estratégias preventivas durante o tratamento, foram identificadas quatro categorias: $\mathrm{EP}^{4,8,9,17,18,19,20,21,22}$

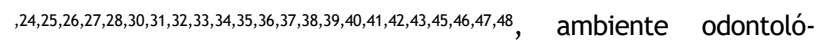
gico $4,8,9,20,22,23,24,28,30,31,32,34,35,36,37,38,39,41,42,43,48$, procedimentos clínicos ${ }^{4,8}$, $9,17,18,19,20,21,22,23,24,25,26,27,28,29,30,32,33,34,35,36,37,38,39,40,41,42,43,46,48$ e cuidados do profissional $4,8,9,17,18,19,20,21,23,24,25,27,28,30,31,32,33,35,37,39,40,41,43,44,45,46,47,48$. $\mathrm{Na}$ categoria EPI, foram citados uso de equipamentos em geral, como: óculos, gorro, máscara cirúrgica, luvas, protetor facial, avental $^{4,8,9,17,18,19,20,21,22,24,25,26,27,28,30,31,32,33,34,35,36,37,38,39,40,41,42,43,45,46,47,48}$ e uso de respirador como N95, FFP2/34,8,17,18,17,20,21,22,24,25,26,27,28,30,31,32,34,35,36,37,38, $39,40,41,42,43,47,48$.

Quanto ao ambiente odontológico foram citados: o uso de sala de pressão negativa ${ }^{4,8,9,20,23,28,30,32,34,35,36,38,42}$, o uso de filtro purificador de ar/HEPA $4,8,31,36,37,38,41,43,48$ e a utilização de barreiras físicas descartáveis ${ }^{23,24,37,39,48}$. Os procedimentos clínicos foram descritos de forma mais detalhada, incluindo o uso de isolamento absoluto $4,8,9,17,18,19,20,21,22,23,25,27,28,29,30,32,33,34,35,36,37,38,39,40,41,42,43,46, \quad$ a aspiração de saliva 4,17,18,19,21,22,23,24,25,27,28,29,30,32,33,35,36,38,39,40,41,43,46,48, evitar produção de aerossol com seringa, ultrassom e alta rotação $8,9,17,18,19,20,21,22,23,24,25,26,27,28,29,30,35,36,38,39,40,41,42,43,46,48$, a preferência por radiografia extraoral ${ }^{9,18,21,22,23,24,27,28,29,32,35,36,37,38,39,43,48}$, a preferência por instrumentos manuais e/ou baixa rotação $8,18,21,22,24,25,27,28,32,33,34,38,39,40,43$, o uso de sistemas antirrefluxo $4,23,27,33,37,38,39,40,41,43,48$, o trabalho a quatro mãos $^{4,19,24,29,32,33,35,38,39}$, o uso de sutura reabsorvível ${ }^{18,28,32,35,36,43,48}$ e evitar procedimentos que induzam vômito ou tosse $8,30,35,37,41$. Quanto aos cuidados do profissional, a higiene das mãos $4,8,9,17$, $18,19,20,21,23,24,25,27,28,30,31,32,33,35,37,39,40,41,43,45,46,47,48$ e a etiqueta respiratória ${ }^{4,30,40,48}$ foram as estratégias citadas com maior frequência.

Por fim, em se tratando das estratégias preventivas após o atendimento, foram identificadas as categorias: cuidados no ambiente odontológico $^{4,8,9,17,18,19,20,22,23,24,25,26,27,28,29,30,31,32,33,34,35,36,37,39,40,41,42,43,44,45,46,47,48}$ 
e cuidados com os materiais e EPI utilizados nos atendimen$\operatorname{tos}^{4,8,17,18,22,24,25,26,27,30,32,33,36,37,39,40,41,43,47,48}$. Os cuidados descritos em relação ao ambiente odontológico foram: a desinfecção das superfícies ${ }^{4,8,9,917,18,19,20,22,23,24,25,26,27,28,29,30,31,32,33,34,35,36,37,39,40,41,42,43,44,45,46,47,48}$, o descarte correto de contaminados $9,18,23,24,25,27,28,31,32,33,36,37,40,43$, a ventilação da sala operatória $9,24,30,31,35,36,39,43,47$ e fechar a sala temporariamente após o atendimento ${ }^{23,25}$. Em relação aos cuidados com materiais e EPI utilizados nos atendimentos, 11 estudos citaram esterilização de instrumentais e cane$\operatorname{tas}^{4,17,24,25,27,30,33,36,37,40,41,43}, 15$ abordaram a desinfecção de materiais reutilizáveis $8,17,18,25,26,27,30,32,33,39,40,41,43,47,48$ e quatro estudos citaram a remoção correta dos EP| $\left.\right|^{4,22,24,37}$.

\section{DISCUSSÃO}

As principais estratégias prévias ao atendimento odontológico encontradas nessa revisão integrativa envolveram: a triagem prévia; a priorização do atendimento de urgências e emergências; os cuidados na sala de espera e algumas medidas de proteção específicas para o paciente, incluindo o uso de enxaguatório, a aferição de temperatura, o uso de EPI, a higienização das mãos, entre outras.

A triagem prévia é de fato muito importante para a identificação de casos suspeitos ou possíveis infectados por COVID-19. Ela pode ser realizada via telefone, questionando se houve contato

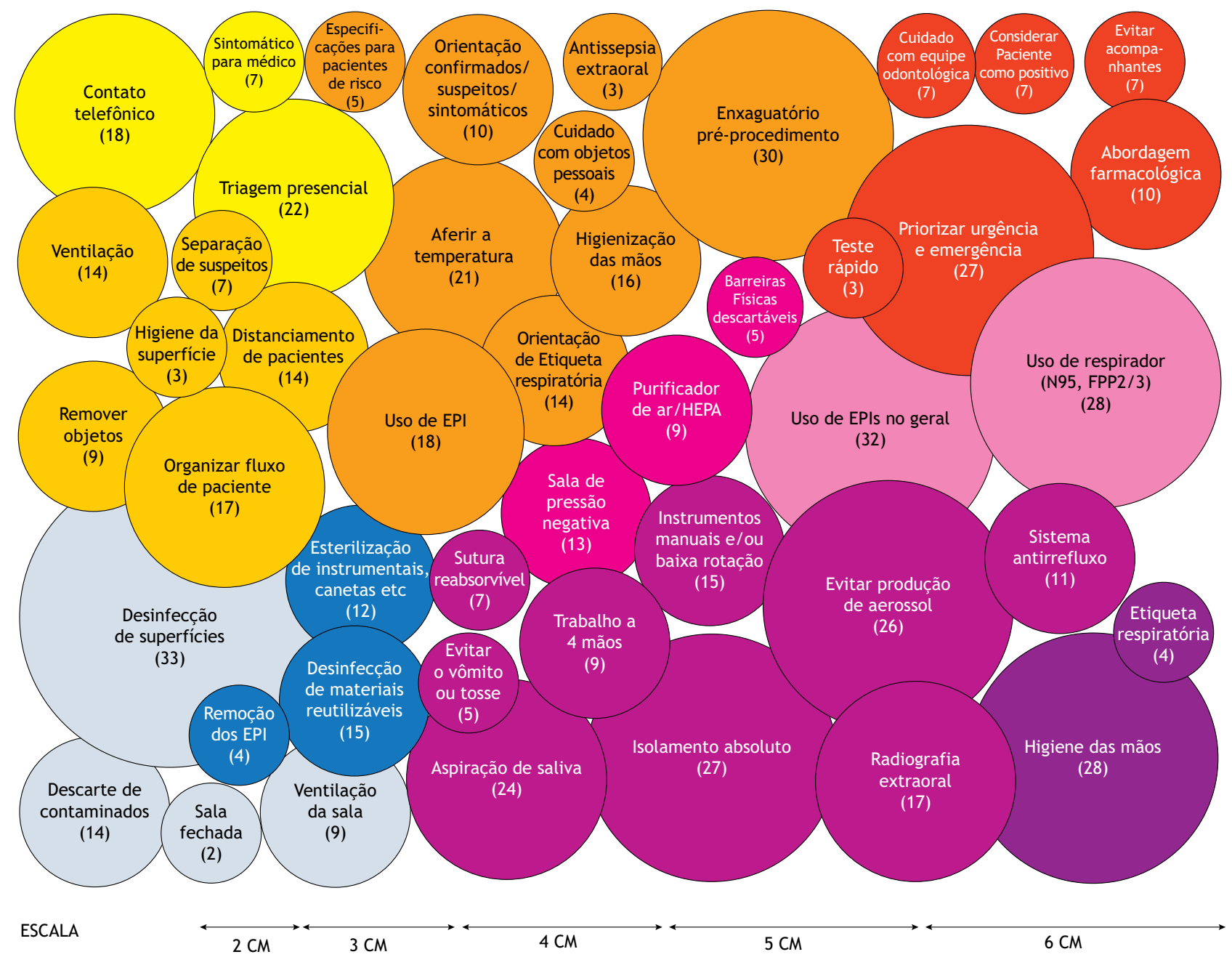

\section{LEGENDA}

Estratégias preventivas antes do atendimento odontológico

Triagem prévia

Cuidados na sala de espera

Medidas de proteção para o paciente

Outros cuidados preventivos
Estratégias preventivas durante 0 atendimento odontológico

EPIs

Ambiente odontológico

Procedimentos clínicos

Cuidados do profissional
Estratégias preventivas após o atendimento odontológico

Cuidados no ambiente odontológico

Cuidados com materiais e EPI utilizados

Fonte: Elaborada pelos autores, 2020

Diâmetro dos círculos: $1-8$ referências $=2 \mathrm{~cm} ; 9-16$ referências $=3 \mathrm{~cm} ; 17-24$ referências $=4 \mathrm{~cm} ; 25-32$ referências $=5 \mathrm{~cm}$, $33-35$ referências $=6 \mathrm{~cm}$.

Figura 2. Estratégias preventivas antes, durante e após o atendimento odontológico. 
com caso positivo ou suspeito; verificando histórico recente de viagens e presença de algum sintoma respiratório como febre ou tosse. Caso alguma das perguntas seja positiva, o atendimento deve ser adiado por pelo menos 14 dias $^{9,27,42}$.

Alguns autores sugeriram questionamento sobre a urgência e a emergência odontológica na qual o paciente se encontra ${ }^{36,39}$. Isso porque, devido à alta propagação do vírus, tem se recomendado o tratamento de pacientes nestas duas circunstâncias, adiando os tratamentos considerados eletivos. Para facilitar essa classificação, a $\mathrm{ADA}^{13}$ emitiu um guia definindo condições de urgência e emergência, conforme descrito na Quadro 2.

Outro aspecto bastante importante é a organização do fluxo de pacientes na clínica odontológica. Recomenda-se limitar o número de indivíduos na sala de espera, espaçando os atendimentos e recebendo apenas um paciente por vez e, quando possível, evitando permitir acompanhantes ${ }^{25,37,41}$.

Na chegada do paciente, este deve preencher uma anamnese detalhada da história médica e responder a um questionário de emergência real ${ }^{9,32}$. A temperatura corporal deve ser aferida, de preferência com um termômetro frontal em que não haja contato com o paciente e, caso este detecte febre acima de $37,5^{\circ} \mathrm{C}$ com ou sem outros sintomas, o atendimento deve ser adiado e o paciente, orientado quanto ao isolamento e à procura de serviço de saúde 4, 9, 10,27,33,39,10.

A grande maioria dos artigos analisados indicou o uso de enxaguatório bucal antes do procedimento, com o objetivo de diminuir a carga viral presente na cavidade bucal, sendo os mais indicados o peróxido de hidrogênio de 0,5 a $1,0 \%$ ou iodopovidona a $0,2 \%, 10,33$. Sabe-se que o SARS-CoV-2 é sensível à oxidação, portanto o mais indicado é o peróxido de hidrogênio ${ }^{33}$. Sobre esse quesito, o Ministério da Saúde divulgou por meio de nota técnica, que o uso de peróxido de hidrogênio deve ser utilizado somente com supervisão do profissional, por haver risco de efeitos colaterais devido ao uso ${ }^{12}$. Alguns estudos também indicam bochecho com clorexidina, cloreto de cetilperidínio e óleos essenciais, porém não há confirmação da eficácia destes enxaguatórios contra o vírus ${ }^{27,46}$.

Os cuidados com a equipe odontológica também foram citados por alguns autores, incluindo: a remoção dos adornos, como brincos, pulseiras, anéis e relógios antes da lavagem das mãos ${ }^{21}$; a manutenção das unhas limpas e a cobertura de cortes com curativos à prova d'água ${ }^{37,48}$; e a aferição da temperatura corporal anteriormente à entrada na clínica ${ }^{10}$.

Além dos cuidados na sala de espera citados no fluxograma, é importante orientar os pacientes sobre etiqueta respiratória, dispondo de alertas visuais como cartazes, placas e pôsteres, além de ressaltar o uso constante de máscara ${ }^{10}$. Segundo nota técnica do Ministério da Saúde ${ }^{12}$, a fim de diminuir a contaminação por microrganismos provenientes do aerossol do atendimento odontológico, as superfícies e bancadas devem estar livres de qualquer material, como prontuários, receituários e objetos pessoais dos pacientes.

Dentre as estratégias preventivas durante os procedimentos está o uso de EPI, tais como: jaleco/avental impermeável, touca, luvas, máscara cirúrgica, óculos, protetores faciais (face shields), roupas e pijamas cirúrgicos, e capas para sapatos ${ }^{10,33}$. Em pesquisa realizada com dentistas da Lombardia na Itália, eles relataram utilizar, entre outros, luvas descartáveis; máscaras cirúrgicas; óculos/viseiras e filtros faciais ${ }^{47}$. Quanto ao uso de respiradores como N95 ou FFP2/3, como analisado na Figura 2, alguns arti$\operatorname{gos}^{21,24,37,43}$ indicaram o uso somente quando há produção de aerossol, e outros ${ }^{17,25,39}$ não especificaram quanto ao momento do uso ou indicaram o uso para a rotina odontológica em geral.

A máscara de proteção respiratória (N95, N99, N100, PFF2 ou PFF3) tem eficácia mínima de filtração de $95 \%$ de partículas de

Quadro 2. Classificação das urgências e emergências odontológicas segundo a American Dental Association.

\begin{tabular}{|c|c|}
\hline Urgência & Emergência \\
\hline 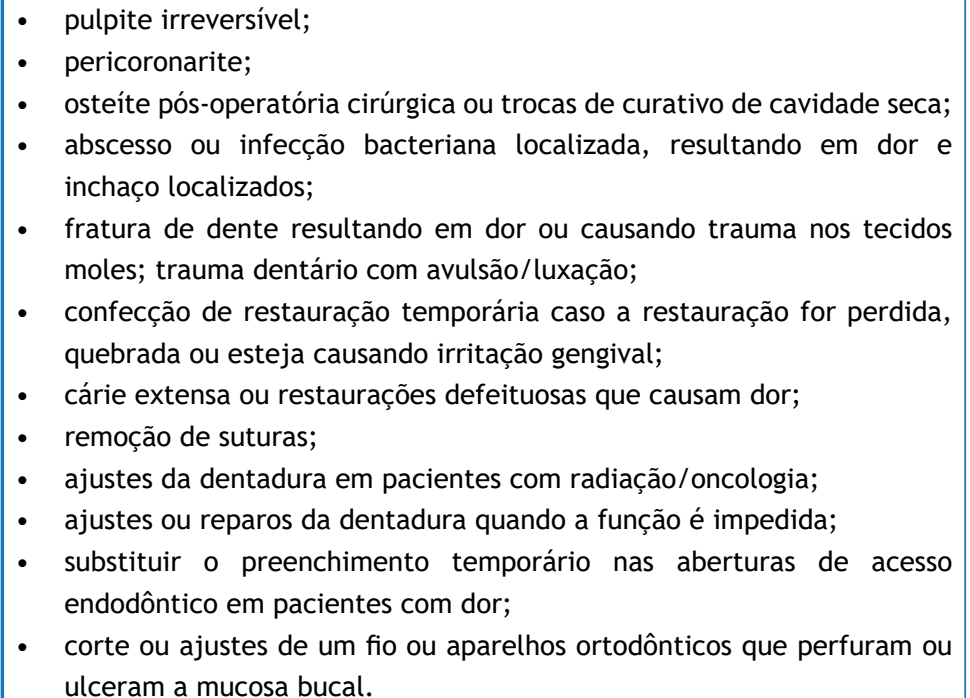 & $\begin{array}{l}\text { - sangramento descontrolado; } \\
\text { moles com edema intrabucal ou extrabucal que } \\
\text { comprometa potencialmente as vias aéreas do } \\
\text { paciente; } \\
\text { trauma envolvendo ossos faciais que potencial- } \\
\text { mente comprometa as vias aéreas do paciente. }\end{array}$ \\
\hline
\end{tabular}

Fonte: $\mathrm{ADA}^{13}$. 
até $0,3 \mu$, e deve estar devidamente ajustada a face do profissional ${ }^{14}$. Estudos recomendaram falar pouco para a N95 não sair do lugar, e os homens devem remover a barba para haver melhor selamento da máscara ${ }^{25,48}$. Deve ainda haver cuidado no armazenamento, manuseio e reutilização dos respiradores 9 . Quanto às estratégias preventivas no ambiente odontológico, alguns artigos citaram atendimento em salas de pressão negativa, que são eficazes na redução dos riscos de transmissão de doenças respiratórias infecciosas, pois há um controle do ar ambiente contaminado $^{20}$. Também indicada pelos autores foi a instalação de filtros de ar (HEPA) no sistema de exaustão que tem por finalidade eliminar contaminantes biológicos do ar exaurido, permitindo a troca de ar de forma constante ${ }^{49}$.

As barreiras mecânicas como filmes de policloreto de vinila (PVC), sacos plásticos e campos de tecido não tecido (TNT) são formas de inibir a infecção cruzada e facilitar a limpeza, porém foram pouco citadas nos artigos. 0 ideal é a colocação desses protetores em locais como: alças de refletores, encostos e braços de cadeiras, canetas de alta rotação, seringa tríplice, pontas de sugadores, além de colocação no mobiliário ${ }^{10,25,37}$.

Quando houver necessidade de fazer o atendimento, o profissional deve dar preferência a procedimentos que não gerem aerossol, utilizando instrumentos manuais e de baixa rotação ${ }^{25,39}$. Da mesma forma, o Ministério da Saúde ${ }^{12}$ recomenda que seja evitado o uso de alta e baixa-rotação, seringa tríplice, jato de bicarbonato e ultrassom, em conformidade com a maioria dos estudos selecionados nesta revisão. Por isso, autores como Franco et al. ${ }^{25}$ e Martins-Chaves et al. ${ }^{23}$ recomendaram que a sala de atendimento deva ser fechada por um tempo para que as partículas sedimentem e, após isso, seja realizada a desinfecção, ou que procedimentos geradores de aerossol sejam deixados para o final do dia ${ }^{35,48}$.

Nos casos em que seja necessário utilizar alta rotação, o uso de isolamento absoluto com aspiração de saliva também foi bastante citado, pois há redução de dispersão de gotículas, secreções e aerossóis durante o procedimento e, sem dúvida, o trabalho a quatro mãos facilita e diminui o tempo de atendimento ${ }^{20,24,32}$. Além disso, Long et al. ${ }^{36}$ sugeriram fazer sucção por 30 seg após cada atendimento. Juntamente, foi recomendado uso de sistemas antirrefluxo, que podem reduzir significativamente o refluxo de bactérias orais nos tubos da peça e da unidade odontológica. As peças sem essa válvula podem aspirar e expulsar os detritos e fluidos durante o procedimento e causar infecção cruzada ${ }^{4,33}$.

As técnicas imaginológicas extraorais, como radiografia panorâmica ou tomografia computadorizada, foram recomendadas por muitos autores, para evitar reflexos de vômito ou tosse, além de diminuir o risco de infecção cruzada por ter menos contato com saliva9,22,28,35.

A lavagem de mãos é uma medida crítica para evitar a disseminação do SARS-CoV-2 e foi muito abordada pelos autores selecionados $^{33,35}$. Os profissionais devem lavar as mãos com água e sabão por um período de 20 a $30 \mathrm{seg}$, antes de examinar os pacientes, antes dos procedimentos, depois de tocar no paciente, depois de tocar nos arredores e equipamentos sem desinfecção e também após tocar na boca, mucosa, pele ou ferida, sangue, fluido corporal e secreção $0^{10,21,33}$. Após a lavagem, pode ser realizada fricção com álcool etílico $70 \% 4,21$.

Como estratégias preventivas após o atendimento odontológico, na quase totalidade dos estudos analisados, a desinfecção de superfícies foi citada. Os agentes descontaminantes e suas concentrações variaram de acordo com os países, porém, de uma forma geral foram indicados: hipoclorito de sódio $0,1 \%$, álcool etílico $70 \%$ e peróxido de hidrogênio $0,5 \%$. Todas as superfícies tocadas devem ser desinfetadas, pois o vírus pode permanecer de 2 a 9 dias nas superfícies ${ }^{15}$. Os resíduos descartáveis gerados durante $o$ atendimento devem ser devidamente descartados e, por serem considerados como infecciosos, armazenados em sacos de dupla camada de cor amarela, e amarrados com ligadura pescoço de ganso $23,27,33$.

Dentre os cuidados com os materiais após o atendimento, alguns autores sugeriram que todos os EPI devem ser retirados antes de sair do consultório, seguindo padronização de desparamentação. Juntamente, deve-se ter o cuidado ao sair da clínica e ao chegar em casa ${ }^{21}$. Foi recomendado higienizar celular, chaves, óculos com álcool 70\%, retirar os sapatos, deixar objetos pessoais em uma caixa na entrada, lavar roupa com alvejante acima de $60^{\circ} \mathrm{C}$ e tomar banho para higienizar as partes mais expostas ${ }^{10,21}$.

De uma forma geral, as medidas de prevenção citadas nos artigos analisados estão de acordo com os manuais, protocolos e notas técnicas, tanto do Ministério da Saúde ${ }^{12}$, quanto do Conselho Federal de Odontologia ${ }^{15}$, e outras instituições competentes.

No presente estudo, houve predominância de artigos do tipo revisão de literatura, em contrapartida foram encontradas poucas pesquisas do tipo quantitativas ${ }^{44,45,46,47}$. Elas avaliaram: se os dentistas continuavam a trabalhar durante a pandemia; se tinham conhecimento suficiente sobre a doença; e a autopercepção do risco associado ao atendimento odontológico diante da COVID-19. Além de avaliar quais as medidas de precaução que os profissionais estavam tomando; os EPI que utilizavam; e qual a atitude diante de um paciente suspeito. Devido à escassez dessas pesquisas, são necessárias mais publicações com o intuito de conhecer a realidade dos dentistas nesse momento.

\section{CONCLUSÕES}

Procurou-se, nesse estudo, sistematizar as principais estratégias descritas na literatura para prevenção de contaminação cruzada durante a pandemia de COVID-19. Os estudos avaliados, embora publicados em diferentes países, apresentaram certo consenso no que se refere às medidas preventivas para o controle da disseminação da COVID-19. Medidas essas que consistem em cuidados na sala de espera, proteção para o paciente, cuidados no ambiente e com os procedimentos odontológicos, uso de EPI e, por fim, a atenção à desinfecção e aos cuidados posteriores ao atendimento.

Os protocolos gerados após o surgimento da doença estão de acordo com os artigos selecionados, trazendo as medidas de prevenção como uma forma de impedir a infecção cruzada antes, 
durante e após o atendimento odontológico. Essas publicações são de extrema importância para orientar os cirurgiões-dentistas, trazendo certa segurança mediante tantas incertezas vivenciadas atualmente.

É importante ressaltar que a maioria dos estudos aqui analisados são classificados como revisão de literatura, de modo que há carência de publicações sobre a temática que analisem a efetividade dessas medidas preventivas, bem como a adesão dos profissionais a tais estratégias. Faz-se necessário, portanto, o desenvolvimento de novas pesquisas que avaliem a percepção dos profissionais acerca do enfrentamento da pandemia e o impacto das medidas de prevenção nos serviços odontológicos.

Observa-se atualização constante dos protocolos publicados, uma vez que novas estratégias estão sendo adotadas de acordo com a evolução do conhecimento sobre o assunto. Esta é também uma limitação desta pesquisa, uma vez que se restringe ao período no qual os dados foram coletados e novas descobertas podem confirmar ou refutar as estratégias aqui descritas.

\section{REFERÊNCIAS}

1. World Health Organization - WHO. Pneumonia of unknown cause: China. Disease Outbreak News. 5 jan 2020[acesso 25 jun 2020]. Disponível em: https://www.who.int/csr/ don/05-january-2020-pneumonia-of-unkown-cause-china/en/

2. Organização Pan-Americana da Saúde - OPAS. Folha informativa: COVID-19 (doença causada pelo novo coronavírus). Washington: Organização Pan-Americana da Saúde; 2020[acesso 25 jun 2020]. Disponível em: https: / / www.paho.org/bra/index.php?option=com_ content\&view $=$ article\&id $=6101$ : covid $19 \& \mid$ temid $=875$

3. Ministério da Saúde (BR). Painel coronavírus. Brasília: Ministério da Saúde; 2020[acesso 25 jun 2020]. Disponível em: https://covid.saude.gov.br/

4. Tuñas ITC, Silva ET, Santiago SBS, Maia KD, Silva Júnior GO. Doença pelo coronavírus 2019 (COVID-19): uma abordagem preventiva para Odontologia. Rev Bras Odontol. 2020;77:1-7. https://doi.org/10.18363/rbo.v77.2020.e1766

5. Ministério da Saúde (BR). O que é COVID-19. Brasília: Ministério da Saúde; 2020[acesso 25 jun 2020]. Disponível em: https://coronavirus.saude.gov.br/sobre-a-doenca

6. Diagnósticos da América - DASA. Coronavírus: tudo sobre a COVID-19. Barueri: Diagnósticos da América; 2020[acesso 25 jun 2020]. Disponível em: https://dasa.com.br/ coronavirus\#lp-pom-block-2856

7. Center for Disease Control and Prevention - CDC. Guidance for dental settings. Washington: Center for Disease Control and Prevention; 2020[acesso 25 jun 2020]. Disponível em: https://www.cdc.gov/coronavirus/2019-ncov/hcp/dentalsettings.html\#PPE

8. Ge ZY, Yang LM, Xia JJ, Fu XH, Zhang YZ. Possible aerosol transmission of COVID-19 and special precautions in dentistry. J Zhejiang Univ Sci B. 2020;21(5):361-8. https: //doi.org/10.1631/jzus.B2010010

9. Ather A, Patel B, Ruparel NB, Diogenes A, Hargreaves KM. Coronavirus disease 19 (COVID-19): implications for clinical dental care. J Endod. 2020;46(5):584-95. https://doi.org/10.1016/j.joen.2020.03.008

10. Conselho Federal de Odontologia - CFO. Manual de boas práticas em biossegurança para ambientes odontológicos. Brasília: Conselho Federal de Odontologia; 2020[acesso 24 jun 2020. Disponível em: https://website.cfo.org.br/wp-content/uploads/2020/04/
cfo-lanc\%cc\%a7a-Manual-de-Boas-Pra\%cc\%81ticas-emBiosseguranc\%cc\%a7a-para-Ambientes-Odontologicos.pdf

11. Associação de Medicina Intensiva Brasileira - AMIB. Conselho Federal de Odontologia - CFO. Recomendações AMIB/CFO para atendimento odontológico COVID-19: comitê de odontologia AMIB/CFO de enfrentamento ao COVID-19. São Paulo: Associação de Medicina Intensiva Brasileira; 2020[acesso 24 jun 2020. Disponível em: https://www.amib.org.br/fileadmin/user_upload/ $\mathrm{amib} / 2020 / \mathrm{marco} / 26 / 2603$ Recomendacoes_AMIB-CFO_ para_atendimento_odontologico_COVID19_atualizada.pdf

12. Ministério da Saúde (BR). Secretaria de Atenção Primária à Saúde. Coordenação-Geral de Saúde Bucal. Nota técnica No 16/2020-AGGSB/DESF/SAPS/ MS. COVID-19 e atendimento odontológico no SUS. Brasília: Ministério da Saúde; 2020[acesso 30 jul 2020]. Disponível em: http: / /www.crosp.org.br/uploads/ arquivo/295c9c14409db20cb63c862bb07ce0e4.pdf

13. American Dental Association - ADA. What constitues a dental emergency? Chicago: American Dental Association; 2020[acesso 25 jun 2020]. Disponível em: https: //success. ada.org/ /media/CPS/Files/Open\%20Files/ADA_COVID19_ Dental_Emergency_DDS.pdf

14. Agência Nacional de Vigilância Sanitária - Anvisa. Orientações para serviços de saúde: medidas de prevenção e controle que devem ser adotadas durante a assistência aos casos suspeitos ou confirmados de infecção pelo novo coronavírus (SARS-CoV-2). Brasília: Agência Nacional de Vigilância Sanitária; 2020.

15. Conselho Federal de Odontologia - CFO. Recomendações para atendimentos odontológicos em tempos de pandemia. Brasília: Conselho Federal de Odontologia; 2020[acesso 25 jun 2020]. Disponível em: https://website.cfo.org.br/ wp-content/uploads/2020/05/Cartilha-cfo-covid19.pdf

16. Landis JR, Koch GG. The measurement of observer agreement for categorical data. Biometrics. 1977;33(1):159-74.

17. Xavier TB, Barbosa GM, Silva BBP, Daroz BG, Santos YP, Conte Neto $\mathrm{N}$ et al. Protocolo de tratamento odontológico na cirurgia e traumatologia buco-maxilo-facial no contexto do COVID-19. Braz J Health Rev. 2020;3(3):4484-500. https://doi.org/10.34119/bjhrv3n3-047 
18. Sepúlveda-Verdugo C, Secchi-Álvarez A, DonosoHofer F. Consideraciones en la atención odontológica de urgencia en contexto de coronavirus COVID-19(SARS-CoV-2). Int JOdontostomat. 2020;14(3):279-84. https://doi.org/10.4067/S0718-381X2020000300279

19. Sales PH, Sales PL, Sales MLH. COVID-2019: how to decrease the risk of infection in dental practice? Minerva Stomatol. 2020:1-10. https://doi.org/10.23736/S0026-4970.20.04372-1

20. Ren YF, Rasubala L, Malmstrom H, Eliav E. Dental care and oral health under the clouds of COVID-19. JDR Clin Trans Res. 2020;20(3):202-10. https://doi.org/10.1177/2380084420924385

21. Pinto LG, Oliveira JJM, Andrade KS, Farias MF, Figueiredo NFD, Romão TCM et al. Recommendations for dental practices during COVID-19 pandemic. Res Soc Develop. 2020;9(7):1-15. https://doi.org/10.33448/rsd-v9i7.4569

22. Pereira LJ, Pereira CV, Murata RM, Pardi V, PereiraDourado SM. Biological and social aspects of Coronavirus Disease 2019 (COVID-19) related to oral health. Braz Oral Res. 2020;34:1-11. https://doi.org/10.1590/1807-3107bor-2020.vol34.0041

23. Martins-Chaves RR, Gomes CC, Gomez RS. Immunocompromised patients and Coronavirus Disease 2019: a review and recommendations for dental health care. Braz Oral Res. 2020;34:1-7. https://doi.org/10.1590/1807-3107bor-2020.vol34.0048

24. Maia ABP, Reis VP, Bezerra AR, Conde DC. Odontologia em tempos de COVID-19: revisão integrativa e proposta de protocolo para atendimento nas unidades de saúde bucal da polícia militar do estado do Rio de Janeiro-PMRJ. Rev Bras Odontol. 2020;77:1-8. https://doi.org/10.18363/rbo.v77.2020.e1812

25. Franco JB, Camargo AR, Peres MPSM. Cuidados odontológicos na era do COVID-19: recomendações para procedimentos odontológicos e profissionais. Rev Assoc Paul Cir Dent. 2020;74(1):18-21.

26. Silva ROC, Zermiani TC, Bonan KFZ, Ditterich RG. Protocolos de atendimento odontológico durante a pandemia de COVID-19 nos países do Mercosul: similaridades e discrepâncias. Vigil Sanit Debate. 2020;8(2):86-9. https: / /doi.org/10.22239/2317-269x.01620

27. Fini MB. What dentists need to know about COVID-19. Oral Oncol. 2020;105:1-5. https://doi.org/10.1016/j.oraloncology.2020.104741

28. Araya-Salas C. Consideraciones para la atención de urgencia odontológica y medidas preventivas para COVID-19(SARS-CoV2). Inter JOdontostomat.2020;14(3):268-70. https://doi.org/10.4067/S0718-381X2020000300268

29. Wu KY, Wu DT, Nguyen TT, Tran SD. COVID-19's impact on private practice and academic dentistry in North America. Oral Dis. 2020:1-4. https://doi.org/10.1111/odi.13444

30. Volgenant CMC, Persoon IF, Ruijter RAG, Soet JJH. Infection control in dental health care during and after the SARS-CoV 2 outbreak. Oral Dis. 2020:1-10. https://doi.org/10.1111/odi.13408
31. Turkistani KA. Precautions and recommendations for orthodontic settings during the COVID-19 outbreak: a review. Am J Orthodont Dent Orthoped. 2020;158(2):175-81. https://doi.org/10.1016/j.ajodo.2020.04.016

32. Shamszadeh S, Parhizkar A, Mardani M, Asgary $S$. Dental considerations after the outbreak of 2019 novel Coronavirus Disease: a review of literature. Arch Clin Infect Dis. 2020;15(2):1-7. https://doi.org/10.5812/archcid.103257

33. Peng X, Xu X, Li Y, Cheng L, Zhou X, Ren B. Transmission routes of 2019-nCoV and controls in dental practice. Inter J Oral Science. 2020;12(1):1-6. https://doi.org/10.1038/s41368-020-0075-9

34. Passarelli PC, Rella E, Manicone PF, GarciaGodoy F, D'Addona A. The impact of the COVID-19 infection in dentistry. Experiment Biol Med. 2020:1-5. https://doi.org/10.1177/1535370220928905

35. Meng L, Hua F, Bian Z. Coronavirus Disease 2019 (COVID-19): emerging and future challenges for dental and oral medicine. J Dent Res. 2020;99(5):481-7. https://doi.org/10.1177/0022034520914246

36. Long RH, Ward TD, Pruett ME, Coleman JF, Plaisance Junior MC. Modifications of emergency dental clinic protocols to combat COVID-19 transmission. Spec Care Dentist. 2020;40(3):219-26. https://doi.org/10.1111/scd.12472

37. Giudice $R$. The severe acute respiratory syndrome coronavirus-2 (SARS-CoV-2) in dentistry: management of biological risk in dental practice. Int J Environ Res Public Health. 2020;17(9):1-12. https://doi.org/10.3390/ijerph17093067

38. Jamal M, Shah MN, Almarzooqi SH, Aber H, Khawaja S, El Abed $R$ et al. Overview of transnational recommendations for COVID-19 transmission control in dental care settings. Oral Dis. 2020:1-10. https://doi.org/10.1111/odi.13431

39. Izzetti R, Nisi M, Gabriele M, Graziani F. COVID-19 transmission in dental practice: brief review of preventive measures in Italy. J Dent Res. 2020;99(9):1030-8. https: / /doi.org/10.1177/0022034520920580

40. Fallahi HR, Keyhan SO, Zandian D, Kim SG, Cheshmi B. Being a front-line dentist during the COVID-19 pandemic: a literature review. Maxillofac Plast Reconstr Surg. 2020;42(1):1-9. https://doi.org/10.1186/s40902-020-00256-5

41. Cirillo N. COVID-19 outbreak: succinct advice for dentists and oral healthcare professionals. Clin Oral Investig. 2020:1-7. https://doi.org/10.1007/s00784-020-03323-3

42. Careddu R, Ciaschetti M, Creavin G, Molina F, Plotino G. COVID-19 and dental practice: overview and protocols during pandemic. G Ital Endodonzia. 2020;34(1):13-19. https://doi.org/10.32067/GIE.2020.34.01.08

43. Bizzoca ME, Campisi G, Muzio LL. COVID-19 pandemic: what changes for dentists and oral medicine experts? A narrative review and novel approaches to infection containment. Int J Environ Res Public Health. 2020;17(11):1-30. https://doi.org/10.3390/ijerph17113793 
44. Gambhir RS, Dhaliwal JS, Aggarwal A, Anand S, Anand V, Bhangu AK. COVID-19: a survey on knowledge, awareness and hygiene practices among dental health professionals in an indian scenario. Rocz Panstw Zakl Hig. 2020;71(2):223-9. https://doi.org/10.32394/rpzh.2020.0115

45. Khader Y, Al Nsour M, Al-Batayneh OB, Saadeh R, Bashier $\mathrm{H}$, Alfaqih $\mathrm{M}$ et al. Dentists' awareness, perception, and attitude regarding COVID-19 and infection control: crosssectional study among jordanian dentists. JMIR Public Health Surveill. 2020.6(2). https://doi.org/10.2196/18798

46. Duruk G, Gümüsşboğa ZS, Çolak C. Investigation of turkish dentists' clinical attitudes and behaviors towards the COVID-19 pandemic: a survey study. Braz Oral Res. 2020;34:1-14. https://doi.org/10.1590/1807-3107
47. Cagetti MG, Cairoli JL, Senna A, Campus G. COVID-19 outbreak in north Italy: an overview on dentistry: a questionnaire survey. Int $\mathrm{J}$ Environ Res Public Health. 2020;17(11):1-12. https://doi.org/10.3390/ijerph17113835

48. Peditto M, Scapellato S, Marcianò A, Costa P, Oteri G. Dentistry during the COVID-19 epidemic: an italian workflow for the management of dental practice. Int J Environ Res Public Health. 2020;17(9):1-15. https://doi.org/10.3390/ijerph17093325

49. Ministério da Saúde (BR). Ações de engenharia em saúde pública para o atendimento de casos de síndrome respiratória aguda grave - SRAG. Brasília: Ministério da Saúde; 2015.

Contribuição dos Autores

Baldan LC, Zermiani TC - Concepção, planejamento (desenho do estudo), aquisição, análise, interpretação dos dados e redação do trabaIho. Teixeira FF - Análise, interpretação dos dados e redação do trabalho. Todos os autores aprovaram a versão final do trabalho.

Os autores informam não haver qualquer potencial conflito de interesse com pares e instituições, políticos ou financeiros deste estudo.

Licença CC BY-NC atribuição não comercial. Com essa licença é permitido acessar, baixar (download), copiar, imprimir, compartilhar, reutilizar e distribuir os artigos, desde que para uso não comercial e com a citação da fonte, conferindo os devidos créditos de autoria e menção à Visa em Debate. Nesses casos, nenhuma permissão é necessária por parte dos autores ou dos editores. 